\title{
CD147 as a Target for COVID-19 Treatment: Suggested Effects of Azithromycin and Stem Cell Engagement
}

\author{
Henning Ulrich ${ }^{1}$ (I) $\cdot$ Micheli M. Pillat ${ }^{2}$ (I) \\ Published online: 20 April 2020 \\ (C) Springer Science+Business Media, LLC, part of Springer Nature 2020
}

\begin{abstract}
The expressive number of deaths and confirmed cases of SARS-CoV-2 call for an urgent demand of effective and available drugs for COVID-19 treatment. CD147, a receptor on host cells, is a novel route for SARS-CoV-2 invasion. Thus, drugs that interfere in the spike protein/CD147 interaction or CD147 expression may inhibit viral invasion and dissemination among other cells, including in progenitor/stem cells. Studies suggest beneficial effects of azithromycin in reducing viral load of hospitalized patients, possibly interfering with ligand/CD147 receptor interactions; however, its possible effects on SARS-CoV-2 invasion has not yet been evaluated. In addition to the possible effect in invasion, azithromycin decreases the expression of some metalloproteinases (downstream to CD147), induces anti-viral responses in primary human bronchial epithelial infected with rhinovirus, decreasing viral replication and release. Moreover, resident lung progenitor/stem are extensively differentiated into myofibroblasts during pulmonary fibrosis, a complication observed in COVID-19 patients. This process, and the possible direct viral invasion of progenitor/stem cells via CD147 or ACE2, could result in the decline of these cellular stocks and failing lung repair. Clinical tests with allogeneic MSCs from healthy individuals are underway to enhance endogenous lung repair and suppress inflammation.
\end{abstract}

Keywords SARS-CoV-2 $\cdot$ Lung stem cells $\cdot$ Coronavirus $\cdot$ Cellular therapy $\cdot$ Asthma $\cdot$ Diabetes mellitus $\cdot$ Invasion $\cdot$ Anti-viral responses $\cdot$ Basigin $\cdot$ EMMPRIN

\section{Introduction}

Currently the world is going through an outbreak of severe acute respiratory syndrome coronavirus 2 (SARS-CoV-2) that can trigger the coronavirus disease 2019 (COVID-19). On March 31, 2020, over 826,000 coronavirus cases, including 40,708 deaths, have already been confirmed around the world, more specifically in 179 countries [1]. These expressive numbers of confirmed cases and death rates call for an urgent demand of effective and available drugs for COVID-19 treatment.

The open-label non-randomized clinical trial of Gautret and co-workers [2] suggested hydroxychloroquine and

Micheli M. Pillat

mmpillat@gmail.com

1 Department of Biochemistry, Institute of Chemistry, University of São Paulo, SP São Paulo, Brazil

2 Department of Microbiology and Parasitology, Health Sciences Center, Federal University of Santa Maria-RS, Avenue Roraima ${ }^{\circ}$ 1000, Santa Maria, RS 97105900, Brazil azithromycin for COVID-19 therapy. In this trial, twenty-six cases were treated daily with $600 \mathrm{mg}$ of hydroxychloroquine (six patients were lost during the monitoring) and presented a significant decrease in viral load after six days compared to untreated controls. Among hydroxychloroquine-treated patients, six also received azithromycin $(500 \mathrm{mg}$ on the first day followed by $250 \mathrm{mg}$ daily) in order to prevent bacterial infection. The most interesting finding of this study was that all patients treated with hydroxychloroquine and azithromycin had negative PCR results in nasopharyngeal samples, i.e., $100 \%$ of patients treated with both drugs were virologically cured compared to $57.1 \%$ of subjects treated with hydroxychloroquine only, and $12.5 \%$ of untreated individuals. Hydroxychloroquine and chloroquine have direct antiviral activity, as demonstrated in vitro [3]. Nine studies have used such drugs for the treatment of patients with COVID-19, including one phase 4 clinical trial (ClinicalTrial.gov Identifier: NCT04316377). Moreover, different action mechanisms of hydroxychloroquine and chloroquine on the COVID-19 have been proposed based on the known action of SARS-CoV-2 and its supposed similarity with the SARS-CoV replication cycle (reviewed by [4]). However, to the best of our 
knowledge, except the clinical trial of Gautret and co-workers [2], there are no other studies and clinical trials investigating the effects of azithromycin in COVID-19. A possible reason for this fact may be that azithromycin is a classical antibiotic with very little effects reported against virus [5-7] and so far unknown effects on SARS-CoV-2 infection.

\section{CD147 as a Target for COVID-19 Treatment}

SARS-CoV-2 invades host cells via two receptors: angiotensin-converting enzyme 2 (ACE2) and CD147 (also known as Basigin or EMMPRIN) [8, 9] (Fig. 1). Spike protein (SP) from virus binds to ACE2 or CD147 on the host cell, mediating viral invasion and dissemination of virus among other cells. The structure of SARS-CoV-2 SP is similar to that of SARS-CoV SP, and both bind to ACE2, invading host cells $[8,9]$. In addition to ACE2, Wang and co-worker [8] recently demonstrated that SARS-CoV-2 SP also binds to CD147. They used Meplazumab, an anti-CD147 humanized antibody, co-immunoprecipitation, ELISA, and immuno-electron microscope to demonstrate the new CD147-SP route of viral invasion. This discovery provides a key target for the development and administration of specific anti-SARS-CoV-2 drugs. A clinical trial in phase II entitled "Clinical Study of Anti-CD147 Humanized Meplazumab for Injection to Treat With 2019-nCoV Pneumonia" (ClinicalTrials.gov Identifier: NCT04275245) is currently underway in China aiming to block CD147 protein by monoclonal antibodies, preventing SARS-CoV-2 SP binding and subsequent infection. This trial is monitored by the virologic clearance rate using Real-Time PCR of respiratory tract samples to evaluate the effects of Meplazumab. This drug is a highly specific molecule directed against CD147, but such a specificity does not exclude that other drugs affecting CD147 expression may also have beneficial effects on COVID-19 treatment.

CD147, known as Basigin or extracellular matrix metalloproteinase inducer (EMMPRIN), was also identified as a red blood cell (RBC) receptor for the parasite Plasmodium falciparum - protozoan that causes Malaria in humans [10]. The complex PfRH5 of the parasite membrane binds CD147 on $\mathrm{RBC}$, and this interaction was essential for invasion by all tested strains in vitro [10]. Experiments with recombinant chimeric antibody (Ab-1) against CD147 also demonstrated that the inhibition of the PfRH5-CD147 interaction potently blocked RBC invasion and cured established infections in a humanized mouse model [11]. Interestingly, azithromycin, which destroys asexual blood-stage parasites by blocking the ribosomal 50S subunit, acts also as an inhibitor of RBC invasion [11-13]. Other macrolide antibiotics were tested and derivatives of azithromycin and erythromycin presented different invasion inhibitory activity [12]. Invasion inhibition assays and experiments of live video microscopy showed rapid azithromycin action to prevent the essential step of tight junction formation, which occurs very early in the invasion process [12]. Removal of two glycan groups of azithromycin induced a remarkable loss of invasion inhibition [12]. In fact, there are some possible mechanisms, by which azithromycin might prevent invasion. A fundamental step in this invasion process is the ligand/receptor interaction [13]. Thus, the interference of azithromycin in PfRH5/CD147 interaction should be considered. If confirmed, this drug might provide a novel therapeutic strategy against other pathogens that invade cells through binding to CD147, such as SARS-CoV-2.

CD147 is a highly glycosylated transmembrane protein of the immunoglobulin super family that acts as the main upstream stimulator of matrix metalloproteinases (MMPs) and may be upregulated in its expression levels during asthmatic and diabetic complications. Expression levels of CD147 and MMPs are often increased in inflammatory processes and tumors and are associated with cancer progression [14]. Cancer stem cells characterized by high cell surface CD147 expression produce more hyaluronan, resulting in stabilization of lipid rafts, as well as reveal enhanced MMP and $A B C$ drug transporter expression, resistance and survival [15]. Recent studies have shown that patients with severe asthma have high levels of MMP-9 in sputum [16], and influenza A virus infection increases CD147 expression in cells from asthmatics [17]. In addition, another study showed that CD147 expression is induced by high glucose $(25 \mathrm{mM})$ concentration in monocytes [18]. High glucose in vitro also increased expression of MMP-1 and MMP-9, and this increase was reverted by suppression of CD147 expression by small interfering RNA and activity inhibition by an anti-CD147 antibody. It is well known that MMPs function in monocyte migration. Thus, inhibition of their regulator CD147 may have beneficial effects in the prevention of diabetic complications involving inflammatory processes [18], including severe acute respiratory syndrome triggers by coronavirus 2. However, specific studies are needed for investigating possible correlations between CD147 and diabetes mellitus in clinical complications due to SARSCoV-2 infection.

Interestingly, doxycycline, an antibiotic analogue of tetracycline, reduced CD147 levels in a gallbladder carcinoma cell line [19] and gingival crevicular fluid (soluble form) in patients with chronic periodontitis [20]. In addition, monocytes and peripheral blood mononuclear cells treated with azithromycin expressed less MMP-9 $[21,22]$, as well as airways of lung transplant patients did upon treatment with azithromycin [23]. Furthermore, MMP-3 and MMP-1 expression was reduced in respiratory epithelial cells in vitro following azithromycin 


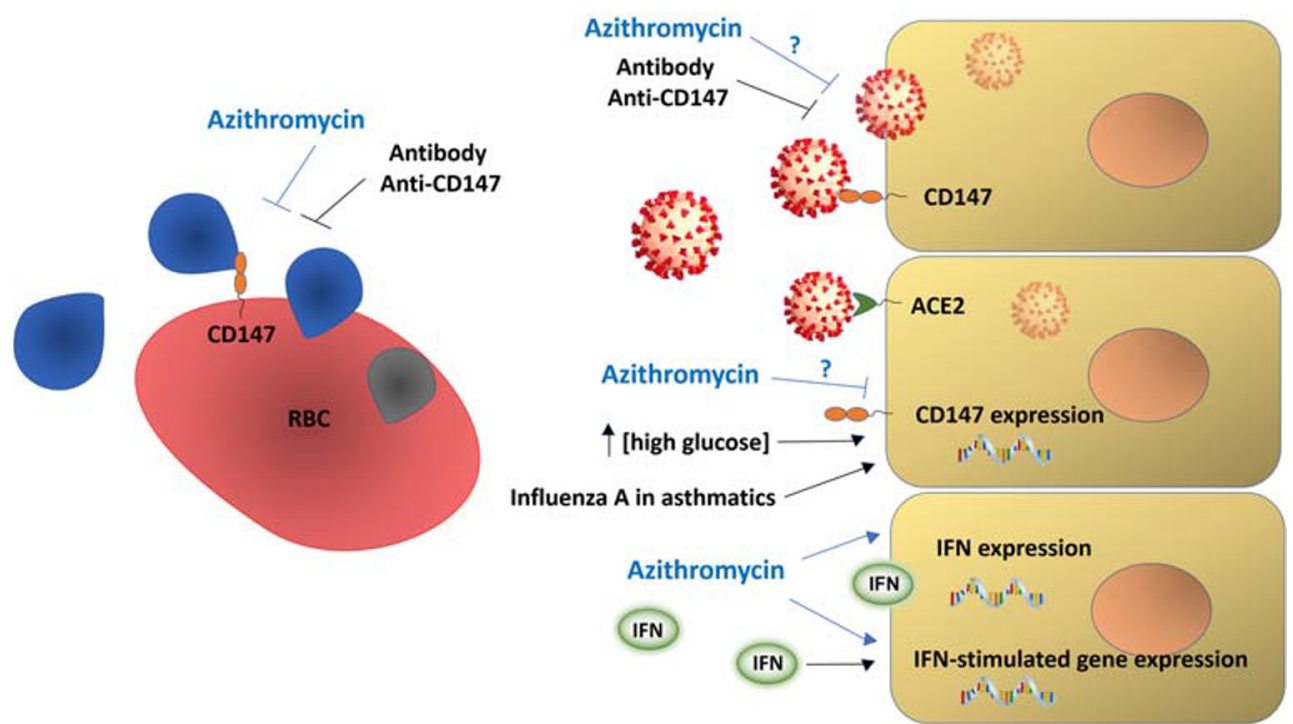

Fig. 1 Targets and possible treatments for COVID-19. CD147 acts as receptor for Plasmodium falciparum (protozoan that causes Malaria) invasion on red blood cells (RBCs). CD147, in addition to ACE2 (angiotensin-converting enzyme 2), also acts as receptor of host cells for SARS-CoV-2 invasion. Treatment with anti-CD147 antibodies prevents invasion of host cells by Plasmodium falciparum and SARS-CoV-2 (in vitro and phase 2 clinical trial). Azithromycin also rapidly prevents Plasmodium falciparum invasion, possibly interfering with vital ligand/ receptor interactions and also SARS-CoV-2 infection. However, the effect of azithromycin on SARS-CoV-2 has not yet been evaluated. Azithromycin induces anti-viral responses in epithelial cells by increasing levels of interferons and interferon-stimulated proteins and decreasing viral replication and virus release. This drug decreases expression of metalloproteinases (MMPs; molecules closely related to CD147). Both, influenza A virus in cells of Asthma patients as well as high glucose concentration in vitro increase CD147 expression, suggesting possible correlations within asthma, diabetes mellitus and CD147 levels in clinical complications due to SARS-CoV-2 infection treatment [24]. In fact, several studies have demonstrated that azithromycin decreases MMP expression and action [21-24]. Despite CD147 and MMPs are closely related molecules, further studies are necessary to evaluate whether azithromycin directly changes expression levels of the CD147 receptor.

Patients with severe COVID-19 present high levels of cytokines in the plasma, specially IL-6, a biomarker for inflammation and robust immune response. In this direction, clinical trials using antibodies anti-IL6 receptors (Sarilumab and Tocilizumab) are already recruited for testing such therapeutic strategy in hospitalized patients with COVID-19. Besides invasion inhibitory activity and antibiotic properties, azithromycin also has immunomodulatory effects, and for this reason has been administrated for the therapy of chronic inflammatory conditions, such as bronchiolitis and rosacea. Although the mechanisms of this anti-inflammatory effect are still little known, some studies presented a reduction of IL-6 levels after azithromycin treatment $[25,26]$. Moreover, a relevant study from Gielen and co-workers [27] demonstrated that azithromycin induces anti-viral responses in primary human bronchial epithelial cells, increasing rhinovirus-induced interferons and interferon-stimulated mRNA and protein expression as well as decreasing rhinovirus replication and release [27].

\section{Stem Cell Facets in COVID-19: Possible Target for Infection, Regeneration, Loss of Stocks and Cellular Therapy}

Stem cells might also be a target for viral infection and interesting therapy for combating COVID-19 lung disease. In a previous seminal work, Ling et al. [28] studied the cellular tropism of SARS-CoV in mouse neonatal Oct-4 positive, SSEA-1, Sca-1, cytokeratin-7 and ACE2 expressing lung cell epithelial colonies surrounded by a stroma of neonatal lung tissue. These residing lung stem cells expressed ACE2, one of the entry receptors of SARS-CoV-2 (Fig. 2). The authors observed Oct- $4^{+}$, long-term BrdU label-retaining cells in the broncho-alveolar junction of the neonatal lung, being in line with the in vitro results. Cultures of Oct-4 lung stem cells could be infected with SARS-CoV with subsequent virus replication, suggesting that failing lung repair of SARS-CoVinfected involves the loss of mobilization and differentiation of lung stem and progenitor cells. On the other hand, CD147, the second entry receptor for SARS-CoV-2, has been proposed to be a marker protein of undifferentiated embryonic stem cells, and the monoclonal antibody against this protein also stained lung cancer cells [29]. It is possible that CD147 is also expressed by untransformed lung stem and progenitor cells; however, to the best of our knowledge, there is no experimental evidence for such affirmation. CD147 is expressed 


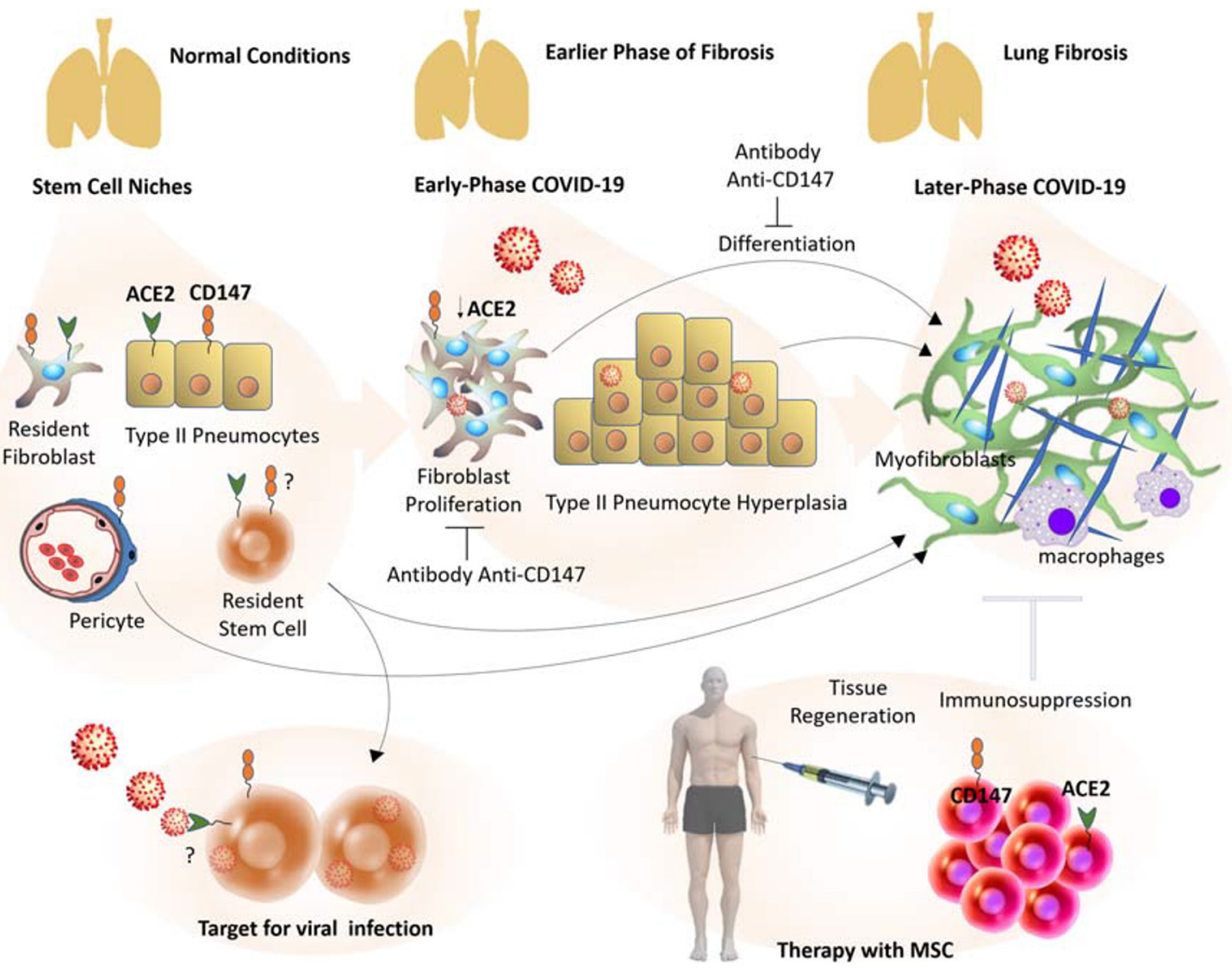

Fig. 2 Stem cells in COVID-19: from a target for viral infection to therapy with mesenchymal stem cells (MSC). The continuous injury to the alveolar zone caused by SARS-CoV-2 infections, possibly origins a pro-inflammatory microenvironment, triggering an aberrant response with partial replacement of normal tissue by fibrous tissue. Pulmonary fibrosis is heavily populated by myofibroblasts. These cells seem to be originated from several types of cells, mainly resident stem cells/MSClike cells. Early-phase COVID-19 pneumonia can show type II pneumocytes hyperplasia and fibroblast proliferation/fibroblast focias

initial step of pulmonary fibrosis, while later COVID-19 phases can present fibrosis and fibrous stripes. Anti-CD147 antibodies inhibit TGF- $\beta 1-$ induced proliferation and differentiation of normal human lung fibroblasts in vitro. In addition to extensive differentiation, possible direct viral invasion of progenitor/stem cells via CD147 or ACE2 could result in the decline of these cellular stocks and failing lung repair. Benefits of cellular therapy with allogeneic MSC transplantation from healthy donators are being investigated. These cells can induce immunosuppression and tissue regeneration

by mesenchymal stem cells of human cord blood and bonemarrow origin, suggesting that the CD147 protein is expressed by tissue-specific stem cells [30]. Thus, besides the loss of airway epithelial cells by viral infection and replication, a second component responsible for missing cellular regeneration is proposed, since stem and regenerating cells can be directly infected and lost.

In addition to direct viral invasion and loss of lung stem cells, stocks of these cells also can be affected during SARSCoV-2 infection by extensive differentiation into myofibroblasts. These cell deposit extracellular matrix proteins and are the main cells presents in fibrotic diseases [31], including pulmonary fibrosis and fibrous stripes due to COVID-19. In fact, current clinical evidences suggest that pulmonary fibrosis or fibrous stripes are important complications due to COVID-19 [32]. Pan and co-workers [33] observed that $17 \%$ of hospitalized COVID-19 patients presented fibrous stripes. Myofibroblasts seem to be originated from several types of cells, such as resident fibroblasts [34], pericytes [35, 36], type II pneumocytes via epithelial-tomesenchymal transition [37], and resident stem cells/MSClike cells $[38,39]$. Recent evidence using lineage tracing showed that resident mesenchymal cells are the main sources of myofibroblasts in pulmonary fibrosis, rather than epithelial or bone marrow-derived cells [40]. It was observed that resident stem cells/MSC-like cells (side population (SP) and $\mathrm{GLI}^{+}$cells) $[39,41]$ play an important role in pulmonary fibrosis, and their numbers decline in mice and patients with this complication compared to healthy controls [38, 39, 41].

ACE2, present in multiple cellular types in lung, including resident stem cells [28], belongs the renin angiotensin system that is involved in pulmonary fibrosis [42, 43]. It was observed that angiotensin-II (Ang-II) is necessary and sufficient to promote fibrosis in mice [42]. On the other hand, ACE2 activity cleaving Ang-II inhibits experimental lung fibrosis, and patients with this clinical complication presented 
downregulated levels of this receptor [42]. CD147, the second entry receptor for SARS-CoV-2, is also present in multiple cellular types in lung and highly expressed in type II pneumocytes and macrophages at the edges of the fibrotic zones [44]. Normal human lung fibroblasts were transiently transfected to overexpress CD147 and presented a significant increase in TGF- $\beta 1$-induced proliferation and expression of $\alpha$-smooth muscle actin, a marker of myofibroblasts. On the other hand, anti-CD147 antibodies inhibited TGF- $\beta 1$-induced proliferation and differentiation of fibroblasts into myofibroblasts [44]. Therefore, the blockade of CD147 could also play a beneficial role in pulmonary fibrosis due to COVID-19.

Proliferating fibroblasts invade the intra-alveolar zone and origin the so called "fibroblast foci". This process is considered the first step in pathogenesis of pulmonary fibrosis [31]. Lung sections from two patients with early-phase COVID-19 pneumonia presented characteristics similar to this initiation step of fibrosis [45]. Fibroblast foci were observed in the airspaces, in addition to edema, type II pneumocyte hyperplasia with inflammatory cellular infiltration and multinucleated giant cells. Some regions of reactive epithelial hyperplasia had also abundant alveolar macrophages [45]. In fact, the continuing injury to the alveolar zone caused by SARS-CoV-2 infections possibly origins a pro-inflammatory microenvironment triggering this aberrant response with partial replacement of normal tissue by fibrous tissue.

Since both early-phase and severe COVID-19 stages show strong involvement of inflammatory components and possibly loss of resident stem cell stocks, a considerable amount of research activities are engaged in investigating the role of cellular therapy using immune response-suppressing MSCs for COVID-19 therapy. In this direction, several clinical trials are planned or already recruited for testing such cellular therapeutic strategy [46]. These trials shall study possible reduction of inflammation and promotion of tissue regeneration following allogeneic MSC transplantation.

\section{Conclusions}

Thus, we have presented here enough evidence to raise some questions: How can the effects of azithromycin in COVID-19 observed by Gautret and co-workers [2] (virologically curing patients in combination with hydroxychloroquine) be explained? Can azithromycin have any invasion inhibitory activity (as already reported elsewhere [11-13]) for the case of COVID-19? Does azithromycin treatment decrease the expression of MMPs and possibly CD147, reducing SARSCoV-2 invasion? Both Plasmodium falciparum causing Malaria and SARS-CoV-2 causing COVID-19 are susceptive to hydroxychloroquine treatment; however, are both pathogens also susceptible to azithromycin because they need of the CD147 protein for cell invasion? Can resident progenitor/stem cells be targets of SARS-CoV-2 infection and/or affected in their differentiation capabilities? Can effects of immunomodulatory and anti-viral response of MCSs and azithromycin, respectively, be therapeutically exploited CODIV-19 treatment? Can patients with asthma or diabetes mellitus have high expression levels of CD147 receptor, and are they for this reason more prone to complications?

We have addressed these issues, highlighting CD147 as a target for COVID-19 treatment and suggesting this protein as a possible pathway for azithromycin-induced effects. Azithromycin has been worldwide used for the treatment of infectious diseases with few side effects and can be rapidly produced in large scale, unlike monoclonal antibodies that generally have a slower production process. Nethertheless, further studies need to be carried out to confirm the possible role and mechanism of the azithromycin in COVID-19. Moreover, progenitor/stem cells can be extensively recruited in pulmonary fibrosis of COVID-19 patients and possibly suffer direct invasion via CD147 or ACE. Thus, besides the loss of airway epithelial cells, a second component of missing cellular regeneration is proposed. Certainly, more basic and clinical investigations into this issue are necessary for effective therapeutic approaches. Combinations of pharmacological and MSC therapy for successful therapy of COVID-19 and other viral diseases might be available in the future.

Acknowledgements This study was supported by Conselho Nacional de Desenvolvimento Científico e Tecnológico (CNPq), Federal University of Santa Maria (UFSM), RS, Brazil. HU acknowledges grant support by Fundação de Amparo à Pesquisa do Estado de São Paulo [São Paulo Research Foundation (FAPESP) Project No. 2018/08426-0] and CNPq.

\section{Compliance with Ethical Standards}

Conflict of Interest The authors declare that there are no conflicts of interest regarding the publication of this paper.

\section{References}

1. Coronavirus Resource Center. (2020). Johns Hopkins University Medicine, https://coronavirus.jhu.edu/. Accessed on: 3/31/2020.

2. Gautret, P., Lagier, J. C., Parola, P., et al. (2020). Hydroxychloroquine and azithromycin as a treatment of COVID19: results of an open-label non-randomized clinical trial. International Journal of Antimicrobial Agents. https://doi.org/10. 1016/j.ijantimicag.2020.105949.

3. Liu, J., Cao, R., Xu, M., et al. (2020). Hydroxychloroquine, a less toxic derivative of chloroquine, is effective in inhibiting SARSCoV-2 infection in vitro. Cell Discovery, 6, 16. https://doi.org/10. 1038/s41421-020-0156-0.

4. Devaux, C. A., Rolain, J. M., Colson, P., et al. (2020). New insights on the antiviral effects of chloroquine against coronavirus: what to expect for COVID-19? International Journal of Antimicrobial Agents. https://doi.org/10.1016/j.ijantimicag.2020.105938. 
5. Retallack, H., Di Lullo, E., Arias, C., et al. (2016). Zika virus cell tropism in the developing human brain and inhibition by azithromycin. Proceedings of the National Academy of Sciences of the United States of America, 113(50):14408-14413. https:// doi.org/10.1073/pnas.1618029113.

6. Madrid, P. B., Panchal, R. G., Warren, T. K., et al. (2015). Evaluation of Ebola Virus Inhibitors for Drug Repurposing. ACS Infect Dis. Jul, 10(7), 317-326. https://doi.org/10.1021/acsinfecdis. 5b00030.

7. Bosseboeuf, E., Aubry, M., Nhan, T., et al. (2018). Azithromycin inhibits the replication of Zika virus. $J$ Antivirals Antiretrovirals, 10(1), 6-11. https://doi.org/10.4172/1948-5964.1000173.

8. Wang, K., Chen, W., Zhou, Y. S., et al. (2020). SARS-CoV-2 invades host cells via a novel route: CD147-spike protein. BioRxiv preprint. https://doi.org/10.1101/2020.03.14.988345.

9. Yan, R., Zhang, Y., Li, Y., et al. (2020). Structural basis for the recognition of the SARS-CoV-2 by full-length human ACE2. Science. https://doi.org/10.1126/science.abb2762.

10. Crosnier, C., Bustamante, L. Y., Bartholdson, S. J., et al. (2011). Basigin is a receptor essential for erythrocyte invasion by Plasmodium falciparum. Nature, 480(7378), 534-537. https://doi. org/10.1038/nature10606.

11. Zenonos, Z. A., Dummler, S. K., Müller-Sienerth, N., et al. (2015). Basigin is a druggable target for hostoriented antimalarial interventions. Journal of Experimental Medicine. https://doi.org/10.1084/ jem.20150032.

12. Wilson, D. W., Goodman, C. D., Sleebs, B. E., et al. (2015). Macrolides rapidly inhibit red blood cell invasion by the human malaria parasite, Plasmodium falciparum. BMC Biology, 13, 52. https://doi.org/10.1186/s12915-015-0162-0.

13. Muralidharan, V., \& Striepen, B. (2015). Teaching old drugs new tricks to stop malaria invasion in its tracks. BMC Biology, 13, 72. https://doi.org/10.1186/s12915-015-0185-6.

14. Kong, L. M., Liao, C. G., Zhang, Y., et al. (2014). A regulatory loop involving miR-22, Sp1, and c-Myc modulates CD147 expression in breast cancer invasion and metastasis. Cancer Research. https://doi. org/10.1158/0008-5472.CAN-13-3555.

15. Toole, B. P. (2019). The CD147-Hyaluronan Axis in Cancer. The Anatomical Record. https://doi.org/10.1002/ar.24147.

16. Mattos, W., Lim, S., Russell, R., et al. (2002). Matrix metalloproteinase-9 expression in asthma: effect of asthma severity, allergen challenge, and inhaled corticosteroids. Chest. https:// doi.org/10.1378/chest.122.5.1543.

17. -Moheimani, F., Koops, J., Williams, T., et al. (2018). Influenza A virus infection dysregulates the expression of microRNA-22 and its targets; CD147 and HDAC4, in epithelium of asthmatics. Respiratory Research. https://doi.org/10.1186/s12931-018-0851-7.

18. Bao, W., Min, D., Twigg, M., et al. (2010). Monocyte CD147 is induced by advanced glycation end products and high glucose concentration: possible role in diabetic complications. American Journal of Physiology. Cell Physiology. https://doi.org/10.1152/ ajpcell.00228.2010.

19. Wang, S., Liu, C., Liu, X., et al. (2017). Effects of matrix metalloproteinase inhibitor doxycycline and CD147 antagonist peptide-9 on gallbladder carcinoma cell lines. Tumor Biology. https://doi.org/ $10.1177 / 1010428317718192$.

20. Emingil, G., Atilla, G., Sorsa, T., et al. (2008). The effect of adjunctive subantimicrobial dose doxycycline therapy on gcf emmprin levels in chronic periodontitis. Journal of Perinatology. https:// doi.org/10.1902/jop.2008.070165

21. Kobayashi, Y., Wada, H., Rossios, C., et al. (2013). A novel macrolide solithromycin exerts superior anti-inflammatory effect via NF-kappaB inhibition. $J$ Pharmacol Exp Ther, 345(1), 76-84. https://doi.org/10.1124/jpet.112.200733.

22. Vandooren, J., Knoops, S., Buzzo, J. L. A., et al. (2017). Differential inhibition of activity, activation and gene expression of MMP-9 in THP-1 cells by azithromycin and minocycline versus bortezomib: A comparative study. PLoS One. https://doi.org/10. 1371/journal.pone.0174853.

23. -Verleden, S. E., Vandooren, J., Vos, R., et al. (2011). Azithromycin decreases MMP-9 expression in the airways of lung transplant recipients. Transpl Immunol. https://doi.org/10.1016/j.trim.2011.06.006.

24. Singh, S., Kubler, A., Utpal, K..et al (2014). Antimycobacterial Drugs Modulate Immunopathogenic Matrix Metalloproteinases in a Cellular Model of Pulmonary Tuberculosis. Antimicrobial Agents and Chemotherapy. https://doi.org/10.1128/AAC.02141-13.

25. Aguai, Z. H., Kode, A., Saslow, J. G., et al. (2007). Azithromycin Suppresses Activation of Nuclear Factor-kappa B and Synthesis of Pro-inflammatory Cytokines in Tracheal Aspirate Cells From Premature Infants. Pediatric Research. https://doi.org/10.1203/ PDR.0b013e318142582d.

26. Bouwman, J. J. M., Visseren, F. L. J., Bouter, P. K., et al. (2004). Azithromycin Inhibits interleukin-6 but Not Fibrinogen Production in Hepatocytes Infected With Cytomegalovirus and Chlamydia Pneumoniae. The Journal of Laboratory and Clinical Medicine, 144(1), 18-26. https://doi.org/10.1016/j.lab.2004.03.012.

27. Gielen, V., Johnston, S. L., \& Edwards, M. R. (2010). Azithromycin induces anti-viral responses in bronchial epithelial cells. The European Respiratory Journal, 36, 646-654. https://oi. org/10.1183/09031936.00095809.

28. Ling, T. Y., Kuo, M. D., Li, C. L., et al. (2006). Identification of pulmonary Oct-4 + stem/progenitor cells and demonstration of their susceptibility to SARS coronavirus (SARS-CoV) infection in vitro. Proceedings of the National Academy of Sciences, 103(25), 9530 9535. https://doi.org/10.1073/pnas.0510232103.

29. Higashi, K., Yagi, M., Arakawa, T., et al. (2015). A novel marker for undifferentiated human embryonic stem cells. Monoclon Antib Immunodiagn Immunother, 34(1), 7-11. https://doi.org/10.1089/ mab.2014.0075.

30. Amati, E., Perbellini, O., Rotta, G., et al. (2018). High-throughput immunophenotypic characterization of bone marrow- and cord blood-derived mesenchymal stromal cells reveals common and differentially expressed markers: identification of angiotensinconverting enzyme (CD143) as a marker differentially expressed between adult and perinatal tissue sources. Stem Cell Research \& Therapy, 16(1), 10. https://doi.org/10.1186/s13287-017-0755-3.

31. - Agha, E. E., Kramann, R., Schneider, R. K., et al. (2017). Mesenchymal Stem Cells in Fibrotic Disease. Cell Stem Cell, 21(2), 166-177. https://doi.org/10.1016/j.stem.2017.07.011.

32. -, J., Wang, J., Wang, B. J., Yang, J. C., et al. (2020). Advances in the Research of Mechanism of Pulmonary Fibrosis Induced by Corona Virus Disease 2019 and the Corresponding Therapeutic Measures. Zhonghua Shao Shang Za Zhi, 36. https://doi.org/10. 3760/cma.j.cn501120-20200307-00132.

33. Pan, Y., Guan, H., Zhou, S., et al. (2020). Initial CT findings and temporal changes in patients with the novel coronavirus pneumonia (2019- nCoV): a study of 63 patients in Wuhan, China. European Radiology. https://doi.org/10.1007/s00330-020-06731-x.

34. Hoyles, R. K., Derrett-Smith, E. C., Khan, K., et al. (2011). An essential role for resident fibroblasts in experimental lung fibrosis is defined by lineage-specific deletion of high-affinity type II transforming growth factor $\mathrm{b}$ receptor. American Journal of Respiratory and Critical Care Medicine, 183, 249-261. https:// doi.org/10.1164/rccm.201002-0279OC.

35. Hung, C., Linn, G., Chow, Y. H., et al. (2013). Role of lung pericytes and resident fibroblasts in the pathogenesis of pulmonary fibrosis. American Journal of Respiratory and Critical Care Medicine, 188, 820-830. https://doi.org/10.1164/rccm.201212-2297OC.

36. - Rock, J. R., Barkauskas, C. E., Cronce, M. J., et al. (2011). Multiple stromal populations contribute to pulmonary fibrosis without evidence for epithelial to mesenchymal transition. Proceedings of the National Academy of Sciences of the United States of 
America, 108, E1475-E1483. https://doi.org/10.1073/pnas. 1117988108

37. - Kim, K. K., Kugler, M. C., Wolters, P. J., et al. (2006). Alveolar epithelial Cell mesenchymal transition develops in vivo during pulmonary fibrosis and is regulated by the extracellular matrix. Proceedings of the National Academy of Sciences of the United States of America 103, 13180-13185. https://doi.org/10.1073/ pnas. 0605669103.

38. - Marriott, S., Baskir, R. S., Gaskill, C., et al. (2014). ABCG2pos lung mesenchymal stem cells are a novel pericyte subpopulation that contributes to fibrotic remodeling. American Journal of Physiology. Cell Physiology, 307, C684-C698. https://doi.org/10. 1152/ajpcell.00114.2014.

39. Kramann, R., Schneider, R. K., DiRocco, D. P., et al. (2015). Perivascular Gli1 + progenitors are key contributors to injuryinduced organ fibrosis. Cell Stem Cell, 16, 51-66. https://doi.org/ 10.1016/j.stem.2014.11.004.

40. Xie, T., Liang, J., Liu, N., et al. (2016). Transcription factor TBX4 regulates myofibroblast accumulation and lung fibrosis. The Journal of Clinical Investigation, 126, 3626. https://doi.org/10. 1172/JCI85328.

41. Jun, D., Garat, C., West, J., et al. (2011). The pathology of bleomycin-induced fibrosis is associated with loss of resident lung mesenchymal stem cells that regulate effector T-cell proliferation. Stem Cells, 29, 725-735. https://doi.org/10.1002/stem.604.
42. Li, X., Molina-Molina, M., Abdul-Hafez, A., et al. (2008). Angiotensin converting enzyme-2 is protective but downregulated in human and experimental lung fibrosis. American Journal of Physiology. Lung Cellular and Molecular Physiology. https://doi. org/10.1152/ajplung.00009.2008.

43. Li, X., Zhang, H., Soledad-Conrad, V., et al. (2003). Bleomycin induced apoptosis of alveolar epithelial cell requires angiotensin synthesis de novo. American Journal of Physiology. Lung Cellular and Molecular Physiology. https://doi.org/10.1152/ ajplung.00273.2002.

44. - Guillot, S., Delaval, P., Brinchault, G., et al. (2006). Increased Extracellular Matrix Metalloproteinase Inducer (EMMPRIN) expression in pulmonary fibrosis. Experimental Lung Research, 32(3-4), 81-97. https://doi.org/10.1080/01902140600710512.

45. Tian, S., Hu, W., Niu, L., et al. (2020). Pulmonary pathology of early-phase 2019 novel coronavirus (COVID-19) pneumonia in two patients with lung cancer. Journal of Thoracic Oncology. https://doi.org/10.1016/j.jtho.2020.02.010.

46. [online] Available at: https://clinicaltrials.gov/ct2/results?cond=COVID$19 \&$ term $=$ stem+cell \&cntry $=\&$ state $=\&$ city $=\&$ dist $=$. NIH U.S. National Library of Medicine. ClinicalTrial.gov. (Accessed 27 Mar 2020).

Publisher's Note Springer Nature remains neutral with regard to jurisdictional claims in published maps and institutional affiliations. 